\title{
Kinetik des Ionentransports durch supramolekulare Kanäle in Einkristallen**
}

\author{
Cyrille D. Assouma, Aurélien Crochet, Yvens Chérémond, Bernd Giese und \\ Katharina M. Fromm*
}

Reaktionen in Einkristallen supramolekularer Anordnungen werden in den meisten Fällen mit dem Zerfall des Kristalls in polykristallines oder amorphes Material in Verbindung gebracht, hervorgerufen durch die Umwandlung und die damit verbundene Deformation während der Reaktion. ${ }^{[1]}$ Die wenigen Fälle, in denen die Umwandlung unter Erhaltung des Einkristalls erfolgt, basieren auf der Sorption/Desorption neutraler Gastmoleküle in robusten dreidimensionalen (3D) Metall-organischen Netzwerken $(\mathrm{MOFs})^{[2]}$ oder dem [2+2]Photodimerisierungsprozess in organischen Verbindungen und Koordinationspolymeren. ${ }^{[3]}$ Ionenaustauschreaktionen, wie sie z.B. für Zeolithe ${ }^{[4]}$ und polykristalline $\mathrm{MOFs}^{[2]}$ berichtet werden, sind oft unvollständig und werden mit Methoden wie Atomabsorptionsspektroskopie und energiedispersive Röntgenspektroskopie charakterisiert. ${ }^{[5]}$ 3DMOFs sind mit Zeolithen verglichen worden. ${ }^{[4]}$ Wir zeigen hier die Bildung eindimensionaler (1D) Kanalsysteme basierend auf Kronenethern, die, ähnlich wie Ionenkanäle in der Biologie $^{[6 a]}$ und synthetische organische Kanäle in Membranen, ${ }^{[6 b-e]}$ dazu in der Lage sind, Alkalimetall-Kationen zu transportieren. Komplexe aus Kronenethern und Alkalimetall-Ionen wurden beschrieben, ${ }^{[7]}$ allerdings kennt man nur sehr wenige Verbindungen, in denen die Bildung eines 1DKanals beobachtet wurde. ${ }^{[7 a-c, e]}$ Hier nutzen wir TrihalogenidAnionen des Typs $\mathrm{X}_{3}^{-}, \mathrm{X}_{2} \mathrm{Y}^{-}$und $\mathrm{XY}_{2}^{-}(\mathrm{X}=\mathrm{I}, \mathrm{Y}=\mathrm{Br})$ als strukturgebende Säulen, welche die stapelförmige Anordnung der Dibenzo-[18]Krone-6(DB[18]C6)-Moleküle induzieren (Abbildung 1). Wir konnten zeigen, dass Einkristalle des so gebildeten Kanalsystems den schnellen Austausch von Alkalimetall-Ionen ermöglichen. Nach unserem Kenntnisstand ist dies das erste Mal, dass die Aktivierungsenergie für den Kationentransport in supramolekularen Kanälen gemessen werden konnte. Die Kristalle verändern weder Form noch Größe unter den experimentellen Bedingungen, was ein Zeichen für ihre große Stabilität ist.

Verbindung 1a ,[ $\left[\mathrm{K}_{4}(\mathrm{DB}[18] \mathrm{C} 6)_{8}\left(\mathrm{H}_{2} \mathrm{O}\right)_{8}\left(\mathrm{I}_{3}\right)_{4}\right]_{n}$ “ (Abbildung 1) wurde in Form von großen stäbchenförmigen Ein-

[*] C. D. Assouma, Dr. A. Crochet, Y. Chérémond, Prof. Dr. B. Giese, Prof. Dr. K. M. Fromm

Department of Chemistry, University of Fribourg

Chemin du Musée 9, 1700 Fribourg (Schweiz)

E-Mail: katharina.fromm@unifr.ch

[*** Die Autoren danken dem Schweizerischen Nationalfonds, der Universität Fribourg und dem FriMat für die großzügige Unterstützung. C.D.A. dankt der Schweizerischen Eidgenossenschaft für ein Stipendium.

D. Hintergrundinformationen zu diesem Beitrag sind im WWW unter http://dx.doi.org/10.1002/ange.201208195 zu finden.

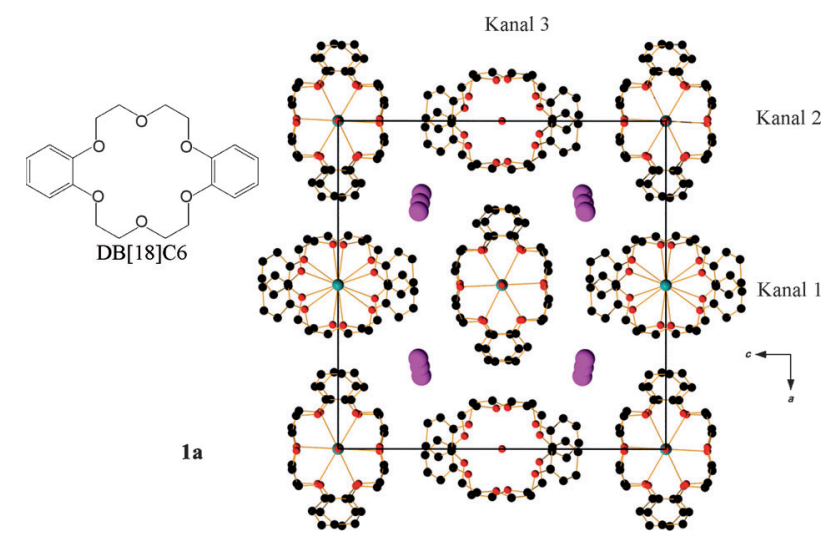

Abbildung 1. DB[18]C6-Molekül (links); Elementarzelle von 1 a entlang $b$, mit zwei C2-Kanälen, einem $\mathrm{Cl}$ - und einem C3-Kanal pro Elementarzelle (rechts); H-Atome wurden weggelassen; schwarz C, rot O, violett I, blau K. Kanal 1 zeigt nach oben, Phenylringe zeigen in Richtung des Lesers oder nach links, Kanal 3 zeigt nach unten oder nach rechts, Kanäle C2 zeigen je einmal nach oben bzw. nach unten.

kristallen (monoklin, $P 2$ ) durch die Reaktion von $\mathrm{KI}_{3}$ mit DB[18]C6 erhalten. ${ }^{[8]}$ Erstaunlicherweise wurden zwischen den quasilinearen Ketten bestehend aus Triiodid-Anionen drei unterschiedliche Arten paralleler und unterschiedlich gefüllter DB[18]C6-Kanäle entlang der $b$-Achse beobachtet (Abbildung 1).

Die erste Art von Kanälen (C1; Abbildung 2) ist mit einer linearen Anordnung aus alternierenden Wassermolekülen und $\mathrm{K}^{+}$-Ionen gefüllt, von denen letztere im Hohlraum der Kronenether zu liegen kommen. In der zweiten Sorte von Kanälen (C2) verbleibt jedes zweite Kronenether-Molekül leer, wohingegen die andere Hälfte mit Kalium-Ionen gefüllt ist, an die in den beiden axialen Positionen je ein Wassermolekül koordiniert. Der letzte Typ von Kanälen (C3) enthält leere, durch Wassermoleküle verbundene DB[18]C6-Einheiten (Abbildung 2, links). In der Elementarzelle ist die Anordnung der Phenylgruppen des DB[18]C6-Liganden in den beiden Kanälen des C2-Typs entgegengesetzt. Diese antiparallele Anordnung, nach unten und oben, wird auch für die beiden anderen Kanäle C1 und C3 beobachtet. Während die Kronenether-Moleküle bezogen auf die Phenylgruppen für den Kanal C2 fast perfekt übereinander liegen, beobachtet man für die Kanäle C1 und C3 einen Versatz der aromatischen Ringe von ca. $20^{\circ}$.

Die zu $1 \mathbf{a}$ isostrukturellen Verbindungen $\mathbf{1 b}$ und $\mathbf{1 c}$, die ebenfalls in der Raumgruppe $P 2$ kristallisieren, wurden durch die Verwendung der Heterotrihalogenide $\mathrm{Br}_{2} \mathrm{I}^{-}$bzw. $\mathrm{BrI}_{2}{ }^{-}$ erhalten. Die Trihalogenide sind alle quasilinear gebaut, jedoch gegenüber der $b$-Achse um ca. $12^{\circ}$ gewinkelt, was eine 


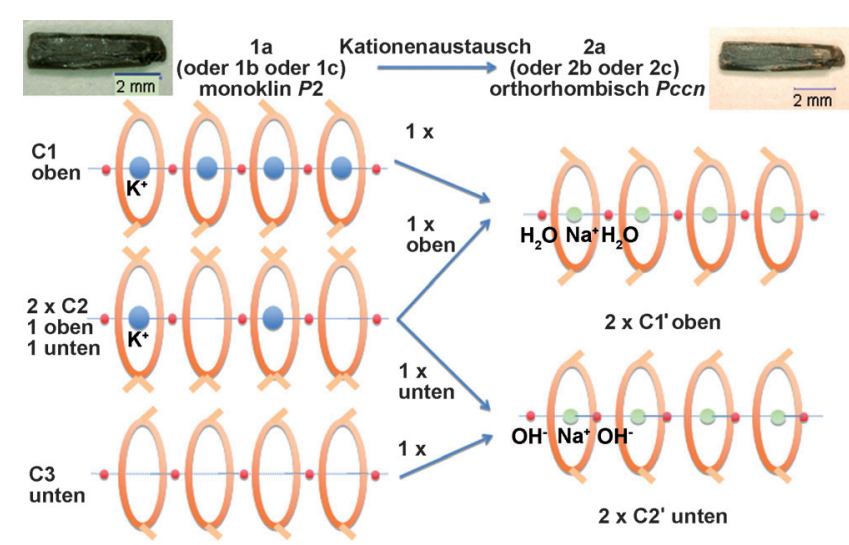

Abbildung 2. Schematische Seitenansicht der drei unterschiedlichen Kanäle in 1 a (bzw. 1 b oder 1 c) mit Andeutung der Orientierung der Phenylgruppen (links), sowie der vorgeschlagenen Umwandlung in $\mathbf{2 a}$ (bzw. $\mathbf{2 b}$ oder $\mathbf{2 c}$ ) (rechts).

Wechselwirkung zwischen dem dritten Halogenatom des einen Anions und dem ersten Halogenatom des nächsten Anions ermöglicht. ${ }^{[9]}$

Basierend auf der stapelförmigen Anordnung der Kronenether und den daraus resultierenden Kanälen, erscheint die Struktur von 1 prädestiniert für den Transport von Kationen. Aus diesem Grund wurden Einkristalle von $1 \mathbf{a}(\mathrm{Er}-$ gebnisse für $\mathbf{1 b}$ in den Hintergrundinformationen) einer $\mathrm{NaOH}-L o ̈ s u n g$ ausgesetzt. So wurde beispielsweise ein $0.37 \times$ $0.1 \times 0.1 \mathrm{~cm}$ großer Einkristall von $\mathbf{1 a}$ für $24 \mathrm{~h}$ in eine $\mathrm{NaOH}-$ Lösung (1M) getaucht, anschließend gewaschen und - äuBerlich unverändert - erneut mittels Röntgendiffraktometrie untersucht. Die Messung ergab die orthorhombische Raumgruppe Pccn mit nahezu unveränderten Zellparametern. Dies ist möglich, weil der $\beta$-Winkel in $\mathbf{1 a}$ sehr nahe an den für das orthorhombische System benötigten $90^{\circ}$ liegt, was wiederum eine sanfte Einkristall-zu-Einkristall-Umwandlung ermöglicht. Die neue Verbindung wurde als $\left[\mathrm{Na}_{2}(\mathrm{DB}[18] \mathrm{C6})_{2}\right.$ $\left.\left(\mathrm{H}_{2} \mathrm{O}\right)(\mathrm{OH}) \mathrm{I}_{3}\right](\mathbf{2 a})$ identifiziert (Abbildung 2) $)^{[10]}$ und enthält zwei unterschiedlich gefüllte Kanäle. $\mathrm{C}^{\prime}$ enthält eine alternierende Kette aus Wassermolekülen und $\mathrm{Na}^{+}$-Ionen, wohingegen $\mathrm{C}_{2}^{\prime}$ eine alternierende Kette aus $\mathrm{HO}^{-}$- und $\mathrm{Na}^{+}$Ionen besitzt. Dies wird durch die Analyse der Bindungslängen für Na1-O7 und Na1-O7' von 2.17(2) bzw. 2.51(2) $\AA$ in $\mathrm{C} 2^{\prime}$ und $\mathrm{Na} 2-\mathrm{O} 8$ von 2.37(2) sowie Na2-O8' von 2.31(2) $\AA$ in $\mathrm{C} 1^{\prime}$ untermauert (siehe Abbildung S15 in den Hintergrundinformationen). Beide Arten von Kanälen verlaufen antiparallel zueinander. Daher ist es wahrscheinlich, dass $\mathrm{C} 1$ und der dazu parallel verlaufende $\mathrm{C} 2-\mathrm{Kanal}$ in $\mathbf{1} \mathbf{a ~} \mathrm{Cl}^{\prime}$ in $\mathbf{2} \mathbf{a}$ bilden, während $\mathrm{C} 3$ und der dazu parallel verlaufende C2-Kanal in $\mathbf{1 a} \mathrm{C}^{\prime}$ in $\mathbf{2}$ a bilden (Abbildung 2). ${ }^{[*]}$

Der Versuch der direkten Synthese des $\mathrm{Na}^{+}$-Analogen $\mathrm{zu}$ 1 a durch das Ersetzen des eingesetzten $\mathrm{KI}_{3}$ mit $\mathrm{NaI}_{3}$ führte zur Bildung von $\left[\mathrm{Na}(\mathrm{DB}[18] \mathrm{C} 6)\left(\mathrm{H}_{2} \mathrm{O}\right)_{2}(\mathrm{DB}[18] \mathrm{C} 6) \mathrm{I}_{3}\right] \quad(\mathbf{3 a}$;

[*] In der Tat ist es wahrscheinlicher, dass sich die Verschiebung der Phenylringe relativ zueinander ändert, als dass die Orientierung der Phenylgruppen während des Transformationsprozesses invertiert wird.
Hintergrundinformationen). Im Gegensatz zu 1a mit drei unterschiedlichen und $\mathbf{2 a}$ mit zwei unterschiedlichen Kanälen, sind in $\mathbf{3 a}$ alle Kanäle gleich gefüllt und enthalten $\mathrm{Na}^{+}$Ionen in der Kavität der Kronenether mit axial je einem gebundenen Wassermolekül. ${ }^{[*]}$ Die Na-O-Abstände für 3 a sind symmetrischer als für 2a und variieren zwischen 2.293(7) und 2.317(7) A. Dennoch gibt es zwei unterschiedliche Arten von Kanälen, die sich lediglich in der Ausrichtung der Phenylringe der DB[18]C6-Kanäle unterscheiden. Analoge Experimente mit $\mathrm{Br}_{2} \mathrm{I}^{-}$bzw. $\mathrm{BrI}_{2}^{-}$als Anion führten zu identischen Ergebnissen (siehe Hintergrundinformationen).

In allen Verbindungen deuten die gestreckten thermischen Ellipsoide der Kationen und Wassermoleküle entlang der Kanalrichtung auf die Mobilität dieser Ionen bzw. Moleküle hin. Aus diesem Grund haben wir die Geschwindigkeit und die Aktivierungsenergie des Ionenaustauschprozesses $\mathbf{1 a}, \mathbf{b} \rightarrow \mathbf{2} \mathbf{a}, \mathbf{b}$ gemessen. Eine wichtige Annahme ist, dass die freie Energie des Ionenaustauschs für monovalente Ionen negativ sein sollte, wenn kleinere Kationen in den Kanal mit hoher Ladungsdichte gelangen. Diese Annahme wurde mit experimentellen Daten für Faujasit ${ }^{[11]}$ untermauert und mit unserem oben beschriebenen Austausch von Kalium- gegen Natrium-Ionen bestätigt. Die Geschwindigkeit des Ionenaustauschs in Membranen und/oder mikroporösen Materialien hängt von unterschiedlichen Parametern ab: Ein Interdiffusionskoeffizient für die ein- und austretenden Kationen berücksichtigt Triebkräfte hervorgerufen durch den Konzentrationsgradienten (Fick) oder Polarisation (Nernst, Planck), die Idealität oder Nichtidealität des Austauschers sowie Wechselwirkungen zwischen Ion-Ion-Flux und Ion-WasserFlux. ${ }^{[12]}$

Kinetische Experimente bezüglich des Ionenaustauschs $\mathbf{1} \rightarrow \mathbf{2}$ in unserem einkristallinen Material wurden mittels Versuchsanordnung A (Abbildung 3) durchgeführt. Der $\mathrm{Na}^{+}$Transport von Kammer a nach $b$ wurde bei zwei unterschiedlichen Temperaturen, $296 \mathrm{~K}$ und $323 \mathrm{~K}$, über einen Zeitraum von $7 \mathrm{~h}$ durch Leitfähigkeitsmessungen untersucht. Zusätzlich wurde die $\mathrm{Na}^{+}$-Konzentration in Kammer b mit optischer Emissionsspektrometrie mit induktiv gekoppeltem Plasma (ICP-OES) bestimmt.

Die Analyse des Ionentransports in $\mathbf{1 a}$ erfolgte nach einer Kinetik pseudo-nullter Ordnung, da die Natrium-IonenKonzentration in Kammer a sehr hoch ist in Bezug auf den niedrigen Wert in Kammer b (Abbildung 3). Die Einkristalle lösten sich nicht auf und blieben während der Messung unverändert in Form und Größe. Die Bestimmung der Geschwindigkeitskonstante $k$ bei zwei unterschiedlichen Temperaturen $\left(k_{1}\right.$ und $\left.k_{2}\right)$ ermöglichte die Berechnung der für den Ionentransport nötigen Aktivierungsenergie $E_{\mathrm{a}}$ mittels der Arrhenius-Gleichung [Gl. (1); $R=$ Gaskonstante, $\mathrm{A}=$ präexponentieller Faktor].

$k=A e^{-\frac{E_{s}}{R T}}$ oder $\ln k=-\frac{E_{\mathrm{a}}}{R T}+\ln A$

[*] Die $\mathrm{Na}^{+}$-Ionen konnten nur an geteilten Positionen platziert werden, sodass jeder Kronenether-Ligand ein halbes Kation beherbergt. Versuche, eine Überstruktur zu identifizieren, in der jede zweite Kronenether-Kavität gefüllt ist, führten nicht zum Erfolg. 


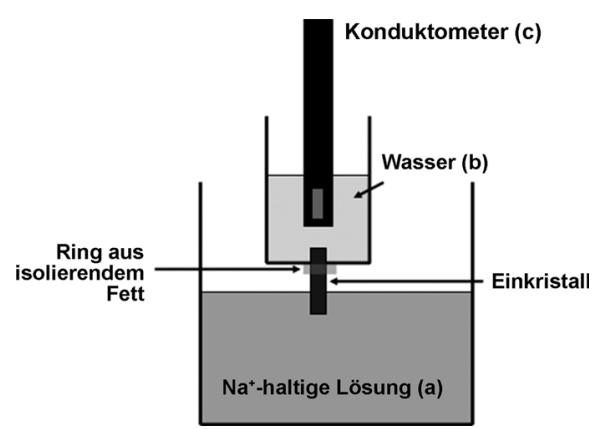

Abbildung 3. Versuchsanordnung $\mathrm{A}$ für die $\mathrm{Na}^{+}$-Leitfähigkeitsmessung.

Verbindungen $\mathbf{1 a}$ und $\mathbf{1 b}$ wurden untersucht, und die kinetischen Experimente des Natrium-Ionentransports lieferten im Rahmen des experimentellen Fehlers die gleichen qualitativen Ergebnisse. Die Zeitabhängigkeit der NatriumIonen-Konzentration in Kammer b bei $296 \mathrm{~K}$ und $323 \mathrm{~K}$ ist exemplarisch für 1b in Abbildung 4 gezeigt (für 1a siehe Hintergrundinformationen). Die Leitfähigkeit nimmt dabei

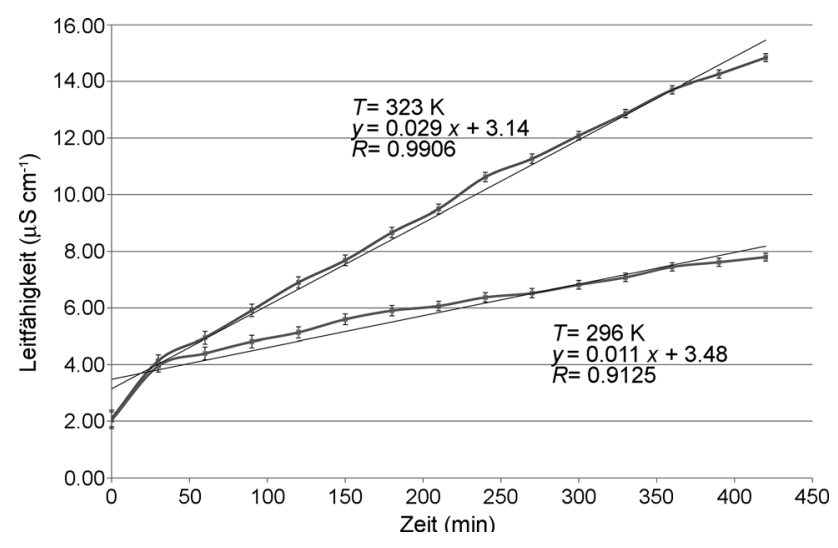

Abbildung 4. Leitfähigkeitsmessung an einem Einkristall $(0.7 \times 0.1 \times 0.1 \mathrm{~cm})$ aus $\left[\mathrm{K}_{4}(\mathrm{DB}[18] \mathrm{C} 6)_{8}\left(\mathrm{H}_{2} \mathrm{O}\right)_{8}\left(\mathrm{Br}_{2} \mathrm{I}\right)_{4}\right]_{n}(1 \mathrm{~b})$. Aufgetragen ist die Zeitabhängigkeit der $\mathrm{Na}^{+}$-Leitfähigkeit bei 296 und $323 \mathrm{~K}$. Fehlerbalken basieren auf drei Messungen.

linear mit der Zeit zu, was in Einklang mit dem Geschwindigkeitsgesetz nullter Ordnung ist. Die Werte wurden korrigiert mit der ionischen Leitfähigkeit, die durch die Messung mit ultrareinem Wasser in beiden Kammern erhalten wurde. Erwartungsgemäß nahm die Leitfähigkeit bei $323 \mathrm{~K}$ schneller zu als bei $296 \mathrm{~K}$. Aus der Steigung (Leitfähigkeit gegen Zeit) ergeben sich für $\mathbf{1 b}$ Geschwindigkeitskonstanten von (1.8 \pm $0.2) \times 10^{-4} \mathrm{molL}^{-1} \mathrm{~s}^{-1}$ bei $296 \mathrm{~K}$ sowie $(4.8 \pm 0.5) \times$ $10^{-4} \mathrm{~mol} \mathrm{~L}^{-1} \mathrm{~s}^{-1}$ bei $323 \mathrm{~K}$. Daraus ergibt sich ein Verhältnis $k_{1} / k_{2}$ von 2.6 \pm 0.4 . Dies wird durch die Messung der NatriumIonen-Konzentration in Kammer b nach $7 \mathrm{~h}$ für beide Temperaturen bestätigt (Tabelle 1). Für Verbindung 1 a wurde auf analoge Weise ein Verhältnis von $2.4 \pm 0.9$ erhalten. Daraus ergibt sich ein Wert für $E_{\mathrm{a}}$ von $(27 \pm 4) \mathrm{kJ} \mathrm{mol}^{-1}$, der lediglich geringfügig höher ist als in superionischen Leitern (10$\left.24 \mathrm{~kJ} \mathrm{~mol}^{-1}\right){ }^{[12]}$ Der schnelle $\mathrm{Na}^{+}$-Transport mittels Wasser durch den Kristall ist in Einklang mit den niedrigen Bin-
Tabelle 1: Konzentration der Natrium-Ionen $\left(\mathrm{mg} \mathrm{L}^{-1}\right)$ in Kammer b nach $7 \mathrm{~h}$ Natriumtransport durch einen Einkristall aus 1 a bzw. 1 b $(0.7 \times 0.1 \times 0.1 \mathrm{~cm})$.

\begin{tabular}{lll}
\hline Verbindung & $296 \mathrm{~K}$ & $323 \mathrm{~K}$ \\
\hline $\mathbf{1 ~ a}$ & $0.30 \pm 0.04$ & $0.48 \pm 0.05$ \\
$\mathbf{1 b}$ & $0.22 \pm 0.04$ & $0.58 \pm 0.01$ \\
\hline
\end{tabular}

dungsaffinitäten der DB[18]C6-Monomere für $\mathrm{Na}^{+}(\log K=$ 1.16) bzw. $\mathrm{K}^{+}(\log K=1.67)^{[13]}$ in Wasser.

Wir interpretieren die Ionenaustauschreaktion $\mathbf{1} \rightarrow \mathbf{2}$ als einen Gleit- oder Hüpfprozess der Ionen durch die Kanäle der Einkristalle mit einer geringen Energiebarriere zwischen den individuellen Kationen-Positionen im Inneren der Kanäle des Kristallgitters.

Interessanterweise ist der $\mathrm{Na}^{+}$-Transport durch den $\mathrm{K}^{+}$enthaltenden Kristall 1 sehr viel schneller als der $\mathrm{K}^{+}$-Transport durch den $\mathrm{Na}^{+}$-enthaltenden Kristall 2. Mit $\mathrm{KOH}(1 \mathrm{M})$ in Kammer a der Versuchsanordnung A (Abbildung 3) wurde eine Geschwindigkeitskonstante für den $\mathrm{K}^{+}$-Transport durch Kristall 2 von lediglich rund $1 \times 10^{-6} \mathrm{molL}^{-1} \mathrm{~s}^{-1}$ bei $296 \mathrm{~K}$ bestimmt (siehe Hintergrundinformationen). Dies lässt sich durch die Tatsache erklären, dass in Verbindung 1 drei unterschiedliche Kanäle in der Elementarzelle vorliegen, von denen einer keine Kationen enthält. Dieser kationenfreie Kanal kann - als initialer Schritt $-\mathrm{Na}^{+}$mit dem sich in der $\mathrm{NaOH}-L o ̈ s u n g$ befindenden Ende binden. Dies muss der Fall sein, da in der finalen Struktur von 2 keine kationenfreien Kanäle auftreten. Nach diesem ersten Komplexierungsprozess von Natrium-Ionen muss die überschüssige positive Ladung eliminiert werden. Dieser Schritt findet am wahrscheinlichsten am anderen Ende des Kristalls durch die Hydration der Ionen mit purem Wasser statt und nicht durch

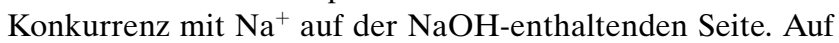
diese Weise wird also ein direkter Ionentransport durch die Kanäle induziert. Der $\mathrm{K}^{+}$-Transport durch 2, der zur Bildung von 1 führt, ist benachteiligt, weil alle Kanäle bereits Ionen enthalten, von denen $25 \%$ von Ionen befreit werden müssten. Der große Unterschied in den Geschwindigkeitskonstanten für den Kationenaustausch (Faktor 180 bei 296 K) lässt sich somit auf die unterschiedlichen Strukturen des $\mathrm{K}^{+}$-enthaltenden Kristalls $\mathbf{1}$ und des $\mathrm{Na}^{+}$-enthaltenden Kristalls 2 zurückführen.

Es stellt sich nun die Frage, ob Einkristalle der Verbindungen 1, 2 oder $\mathbf{3}$ in der Lage sind, auch Lithium-Ionen zu leiten. Das Einwirken von LiI oder LiOH auf die Einkristalle, entweder durch direktes Eintauchen oder unter Benutzung des Versuchsaufbaus A, führte jedoch in allen Fällen zum Zerfall des Kristalls in ein weißes Puder, das zu größten Teilen als LiI und Kronenether identifiziert werden konnte. Dies liegt hauptsächlich daran, dass das kleine Kation $\mathrm{Li}^{+}$die Trihalogenide, und damit die stützenden Säulen, destabilisiert, welche wahrscheinlich nötig sind, um die Gerüststruktur im Festkörper zu erhalten. Werden diese Säulen zerstört, kommt es nicht zu einer Stapelung der Kronenether-Moleküle, und das supramolekulare Gebilde fällt auseinander.

Fazit: Trihalogenid-Ionen können als strukturelle Säulen für die Bildung dreier unterschiedlich gefüllter Kanäle dienen, die auf DB[18]C6 basieren. Der $\mathrm{K}^{+}$-enthaltende 
Einkristall des Typs 1 besitzt ein robustes Untergitter aus gestapelten Kronenether-Molekülen für den $\mathrm{Na}^{+}$-Transport. Kinetische Experimente bei unterschiedlichen Temperaturen zeigen, dass diese supramolekularen Kristalle hervorragende Ionenleiter sind.

Eingegangen am 11. Oktober 2012,

veränderte Fassung am 14. Januar 2013

Online veröffentlicht am 25. März 2013

Stichwörter: Alkalimetalle · Halogenbindung · Ionenkanäle lonentransport · Kronenether

[1] a) G. J. Halder, C. J. Kepert, Aust. J. Chem. 2006, 59, 597-604 b) S. Takahashi, H. Miura, H. Kasai, S. Okada, H. Oikawa, H. Nakanishi, J. Am. Chem. Soc. 2002, 124, 10944-10945.

[2] Zum Beispiel: a) H. J. Park, D.-W. Lim, W. S. Yang, T.-R. Oh, M. P. Suh, Chem. Eur. J. 2011, 17, 7251-7260; b) M.-S. Chen, M. Chen, S. Takamizawa, T. Okamura, J. Fan, W.-Y. Sun, Chem. Commun. 2011, 47, 3787-3789; c) T. Jacobs, J.-A. Gertenbach, D. Das, L. J. Barbour, Aust. J. Chem. 2010, 63, 573-577; d) S. M. Mobin, A. K. Srivastava, P. Mathur, G. K. Lahiri, Dalton Trans. 2010, 39, 1447-1449; e) S. M. Mobin, A. K. Srivastava, P. Mathur, G. K. Lahiri, Inorg. Chem. 2009, 48, 4652-4654;f) B. Li, R.-J. Wei, J. Tao, R.-B. Huang, L.-S. Zheng, Z. Zheng, J. Am. Chem. Soc. 2010, 132, 1558-1566; g) M.-L. Cao, H.-J. Mo, J.-J. Liang, B.-H. Ye, CrystEngComm 2009, 11, 784-790; h) S. M. Neville, G. J. Halder, K. W. Chapman, M. B. Duriska, P. D Southon, J. D. Cashion, J.-F. Létard, B. Moubaraki, K. S. Murray, C. J. Kepert, J. Am. Chem. Soc. 2008, 130, 2869-2876; i) C.-D Wu, W. Lin, Angew. Chem. 2005, 117, 1994-1997; Angew. Chem. Int. Ed. 2005, 44, 1958-1961; j) J. Zhao, L. Mi, J. Hu, H, Hou, Y. Fan, J. Am. Chem. Soc. 2008, 130, 15222-15223.

[3] a) D. Liu, Z.-G. Ren, H.-X. Li, J.-P. Lang, N.-Y. Li, B. F. Abrahams, Angew. Chem. 2010, 122, 4877-4880; Angew. Chem. Int. Ed. 2010, 49, 4767-4770; b) Q. Chu, D. C. Swenson, L. R. MacGillivray, Angew. Chem. 2005, 117, 3635-3638; Angew. Chem. Int. Ed. 2005, 44, 3569-3572; c) J. B. Benedict, P. Coppens, J. Phys. Chem. A 2009, 113, 3116-3120.

[4] Zum Beispiel:G. Ferey in From Zeolites to Porous MOF Materials (Eds: R. Xu, Z. Gao, J. Chen, W. Yan), Elsevier, Amsterdam, 2007, S. 66-84.

[5] a) W.-G. Lu, L. Jiang, X.-L. Feng, T.-B. Lu, Inorg. Chem. 2009, 48, 6997-6999; b) J. An, N. L. Rosi, J. Am. Chem. Soc. 2010, 132 5578-5579; c) G. Calleja, J. A. Botas, M. Sanchez-Sanchez, M. G. Orcajo, Int. J. Hydrogen Energy 2010, 35, 9916-9923; d) S. $\mathrm{Liu}, \mathrm{J} . \mathrm{Li}, \mathrm{F}$. Luo, Inorg. Chem. Commun. 2010, 13, 870-872.
[6] a) „Biological Membrane Ion Channels-Dynamics, Structure and Applications": Biological and Medical Physics, Biomedical Engineering (Hrsg.: S.-H. Chung, O. S. Anderson, V. V. Krishnamurthy), Springer, Berlin, 2007, DOI: 10.1007/0-387-689192; b) J. K. W. Chui, T. M. Fyles, Chem. Soc. Rev. 2012, 41, $148-$ 175 ; c) S. Matile, A. Vargas Jentzsch, J. Montenegro, A. Fin, Chem. Soc. Rev. 2011, 40, 2453-2474; d) G. W. Gokel, N. Berkey, New J. Chem. 2009, 33, 947 -963; e) P.-L. Boudreault, N. Voyer, Org. Biomol. Chem. 2007, 5, 1459-1465.

[7] a) R. D. Bergougnant, K. M. Fromm, Solid State Sci. 2007, 9, 580-587, zit. Lit.; b) K. M. Fromm, E. D. Gueneau, H. Goesmann, C. G. Bochet, Z. Anorg. Allg. Chem. 2003, 629, 597-600; c) R. D. Bergougnant, A. Y. Robin, K. M. Fromm, Cryst. Growth Des. 2005, 36, 4548-4550; d) R. D. Bergougnant, A. Y. Robin, K. M. Fromm, Tetrahedron 2007, 63, 10751-19757; e) L. Kloo, P. H. Svensson, M. J. Taylor, J. Chem. Soc. Dalton Trans. 2000, $1061-1065$

[8] Chekhlov erhielt unter denselben Bedingungen eine Verbindung, die er als $\left[\mathrm{K}(\mathrm{DB}[18] \mathrm{C} 6)\left(\mathrm{H}_{2} \mathrm{O}\right)\right] \mathrm{I}_{3}$ mit einer orthorhombischen Raumgruppe Pccn beschrieb, was wir nicht bestätigen konnten. A. N. Chekhlov, Zh. Neorg. Khim. 2009, 54, 516-520. Auch bei uns wurde beim Lösen der Struktur von 1a die orthorhombische Raumgruppe vorgeschlagen, jedoch lieferte die monokline Raumgruppe die besseren Ergebnisse. Außerdem führte vorsichtiges Erwärmen des Einkristalls 1a auf $180^{\circ} \mathrm{C}$ für $2 \mathrm{~h}$ zur Bildung des wasserfreien Kristalls $\mathbf{1 a}^{\prime}$, [\{KI(DB[18]C6) $\left.\}_{2}(\mathrm{DB}[18] \mathrm{C} 6)\right]$ (monoklin, $P 2_{1} / n$, Hintergrundinformationen), welcher sowohl leere als auch $\mathrm{K}^{+}$-gefüllte DB[18]C6-Kanäle enthält und damit indirekt das Vorhandensein dreier Kanaltypen in $\mathbf{1 a}$ bestätigt.

[9] P. Metrangolo, G. Resnati, Chem. Eur. J. 2001, 7, 2511-2519.

[10] Der Ursprung der Elementarzelle für 2a bildet nun das zentrale Halogenatom des Trihalogenids, während für $\mathbf{1} \mathbf{a}$ das $\mathrm{K}^{+}$-Ion den Ursprung bildete.

[11] a) R. P. Townsend in Introduction to Zeolite Science and Practice (Hrsg.: H. van Bekkum, E. M. Flanigen, J. C. Jansen), Elsevier, Amsterdam, 1991, S. 359-391; b) R. M. Barrer, R. F. Bartholomew, L. V. C. Rees, J. Phys. Chem. Solids 1963, 24, 51-62; c) M. Barrer, L. V. C. Rees, J. Phys. Chem. Solids 1964, 25, 1035-1038; d) S. C. Duffy, L. V. C. Rees, J. Chromatogr. 1974, 102,149-153; e) S. Chaudhury, A. Bhattacharya, C. Agarwal, A. Goswami, J. Phys. Chem. B 2011, 115, 9395 -9400; f) K. E. Doan, B. J. Heyen, M. A. Ratner, D. F. Shriver, Chem. Mater. 1990, 2, 539-545.

[12] a) T. Okura, M. Saimaru, H. Monma, K. Yamasita, Solid State Ionics 2009, 180, 537-540; b) A. L. Despotuli, A. V. Andreeva, B. Rambabu, Ionics 2005, 11, 306-314.

[13] R. M. Izatt, J. S. Bradshaw, S. A. Nielsen, J. D. Lamb, J. J. Christensen, D. Sen, Chem. Rev. 1985, 85, 271-339. 